\title{
Labyrinthe
}

18 | 2004 (2)

La Recherche dans tous ses éclats

\section{Tentative pour une histoire de la globalisation}

«La fabrique de l'universel » Réseaux supranationaux et question urbaine au $\mathrm{xx}^{\mathrm{e}}$ siècle, un terrain d'histoire transnationale et ses développements.

Séminaire complémentaire de l'EHESS dirigé par Pierre-Yves Saunier

\section{Clément Orillard}

\section{(2) OpenEdition}

\section{Journals}

Édition électronique

URL : http://journals.openedition.org/labyrinthe/218

DOI : 10.4000/labyrinthe. 218

ISSN : $1950-6031$

Éditeur

Hermann

Édition imprimée

Date de publication : 15 juillet 2004

Pagination : 69-73

\section{Référence électronique}

Clément Orillard, «Tentative pour une histoire de la globalisation », Labyrinthe [En ligne], 18 | 2004 (2), mis en ligne le 24 juin 2008, consulté le 01 mai 2019. URL : http://journals.openedition.org/ labyrinthe/218 ; DOI : 10.4000/labyrinthe.218 


\title{
TENTATIVE POUR UNE HISTOIRE DE LA GLOBALISATION
}

\author{
«La fabrique de l'universel »* \\ Séminaire complémentaire de l'EHESS \\ dirigé par Pierre-Yves Saunier
}

Clément ORILLARD

orillard@aol.com

Deux fois par mois, dans une salle de l'annexe de l'École des hautes études en sciences sociales ${ }^{1}$, se réunit un petit groupe. Thème de travail: «La fabrique de l'universel». Malgré cet intitulé quelque peu cryptique, point de banderoles alter-mondialistes entreposées dans un angle de la pièce, point de complot néo-libéral ourdi en secret. La globalisation, notion qui rencontre un succès jamais démenti depuis une dizaine d'années, est bien le sujet qui rassemble les membres de ce groupe. Mais ici, les propos échangés traitent de son histoire, abordée à travers une entrée clairement identifiée : la question urbaine au $\mathrm{XX}^{\mathrm{e}}$ siècle.

Première interrogation qui vient à l'esprit du lecteur: pourquoi aborder l'histoire de la globalisation du point de vue urbain ? Pour trouver une réponse, attardons-nous sur le parcours du directeur de ce séminaire, Pierre-Yves Saunier ${ }^{2}$. Spécialisé à l'origine dans l'histoire urbaine contemporaine de Lyon, il s'est plus particulièrement intéressé aux milieux municipaux et civiques lyonnais entre 1900 et 1940 avant de s'interroger sur les réseaux auxquels participaient ces milieux et les structures internationales les organisant : associations, organisations intergouvernementales, etc. ${ }^{3}$ La problématique du séminaire reproduit fidèlement ce triple intérêt, construit le long d'un cheminement de chercheur, en s'articulant autour de trois pôles.

\footnotetext{
* Réseaux supranationaux et question urbaine au $\mathrm{XX}^{\mathrm{e}}$ siècle, un terrain d'histoire transnationale et ses développements.

1. Salle 10, 105 bd Raspail, premier et troisième jeudis du mois de 12 à 14 heures.

2. Chargé de recherche au CNRS, UMR 5600, «Environnement ville et société », université Lyon III.

3. Voir Pierre-Yves Saunier, «Changing the City : Urban International Information and the Lyon Municipality, 1900-1940» dans Planning Perspectives, vol. 14, n 1 , janvier 1999, p. 19-48.
} 
Le premier porte sur les questions urbaines dans la période contemporaine. Mais la ville dont il est question ici est une ville bien particulière. Il ne s'agit pas de celle des pratiques et des représentations sociales, des discours savants scientifiques ou littéraires, mais de celle des politiques et des administrations publiques, question qui en ellemême excède le terrain strictement urbain. Enfin, cette ville particulière n'est pas mise en scène à travers les structures localisées qui peuvent l'animer; au contraire, le regard se porte sur les organisations internationales qui ont assuré et assurent toujours un transfert au niveau mondial de ces questions ${ }^{4}$. Nous voici donc confrontés à une problématique historique qui gravite autour de trois entrées : la ville contemporaine, les politiques et administrations publiques, la mondialisation. Leur articulation est, néanmoins, délibérément orientée dans un sens particulier. Il ne s'agit pas de proposer une histoire mondiale des questions urbaines, mais plutôt d' «historiciser la globalisation» à partir de l'entrée urbaine.

Pour Pierre-Yves Saunier, en effet, «le terrain urbain semble être une bonne occasion pour interroger un des moments historiques où divers acteurs, structures, agents s'affrontent et se rejoignent dans le travail de définition de normes, de pratiques à vocation universelle, participant ainsi à l'écriture théorique et pratique de "l'utopie planétaire". Il y a là une occasion de participer à ce qui serait finalement une histoire de la globalisation, autour du processus de constitution d'un référent transnational au $\mathrm{XX}^{\mathrm{e}}$ siècle, avec ses acteurs, contraintes, projets, limites, échecs ».

Ici intervient une seconde interrogation : comment « historiciser» la globalisation ? La réponse se situe à rebours du parcours scientifique de Pierre-Yves Saunier. Il ne s'agit pas de partir du local mais d'un angle d'approche mondial, à travers une démarche historique «transnationale». Ce caractère «transnational» est identifié comme expérimental et constitue le versant méthodologique de la recherche développée dans ce séminaires.

4. Cette approche des questions urbaines se situe dans le prolongement des travaux qu'a pu développer Christian Topalov, par ailleurs «parrain » de ce séminaire, sur les « mondes de la réforme urbaine. » Voir Christian Topalov, Laboratoires du nouveau siècle. La nébuleuse réformatrice et ses réseaux en France 1880-1914, Paris, éditions de l'EHESS, 1999.

5. Comme exemple d'histoire transnationale basée sur l'idée d' " universel », voir Pascale Casanova, La République mondiale des lettres, Paris, Seuil, 1999. 
Ainsi est-il indiqué dans le programme du séminaire qu'il s'agira d'interroger plus généralement «les limites, les leurres et les richesses d'une posture "transnationale" » en terme de méthodes et d'outils, et de «réfléchir aux possibilités ouvertes par un tel angle au regard des pratiques de l'histoire comparative». Au sein de cette histoire «transnationale», la métaphore utilisée pour décrire la construction de cette «globalisation» qui s'étale dans le temps long est celle de la «fabrique», une «fabrique de l'universel».

Comment la caractériser? La reconstruction de son "économie», c'est-à-dire des lieux, des moyens mobilisés et des structures qui l'ont encadrée est une première piste, qui fut suivie durant la première année et le début de la seconde année de ce séminaire.

La question des lieux s'est essentiellement posée en terme de «foyers » où aurait émergé cette pensée urbaine administrative et politique globale sous le signe de la rationalité et à partir desquels elle se serait diffusée. Deux de ces «foyers» se sont distingués dès les premières séances: Chicago aux États-Unis et Bruxelles en Europe. Plus complexe a été la question des moyens mobilisés dans la diffusion de la pensée administrative et politique étudiée. Parmi le bestiaire varié formé par ces «vecteurs» de la globalisation urbaine, trois groupes ont été identifiés en fonction de leur nature.

Ainsi retrouvons-nous non seulement des objets comme les revues, des événements donnant lieu à des interactions entre acteurs, comme les expositions et les congrès, mais aussi des acteurs, nommés «entrepreneurs de l'universel», qui se sont attachés par eux-mêmes à construire cette diffusion.

Enfin, en ce qui concerne la reconnaissance des structures qui ont encadré cette globalisation urbaine, trois nouvelles catégories émergent: les associations internationales, qui deviennent par la suite organisations non-gouvernementales, les organisations intergouvernementales et les fondations philanthropiques.

C'est à partir de la reconstruction de cette «économie» qu'un second niveau de lecture est apparu. Dès la première année du séminaire, la «géographie» de cette «fabrique» s'est donnée à voir à travers les liens et les tensions associant les éléments identifiés. Ainsi Bruxelles, comme «foyer», est-elle attachée à la naissance du mouvement des associations internationales qui ont essentiellement utilisé les expositions et les congrès animés par les premiers «entrepreneurs de 
l'universel ${ }^{6} »$, alors que Chicago a été le principal lieu de naissance de la pensée qui allait animer les fondations philanthropiques. Ces dernières furent par ailleurs presque toutes américaines et s'appuyèrent moins sur des vecteurs non-humains que sur des individus, ces «entrepreneurs de l'universel» qui parcourent le monde dans un objectif de réforme ${ }^{7}$. À travers cette «géographie», une tension apparaît clairement entre deux pôles: le premier s'appuie sur l'internationalisme animant l'Europe du début du $\mathrm{XX}^{\mathrm{e}}$ siècle, tandis que le second est marqué par une pensée plus universaliste et lié aux fondations philanthropiques américaines. Entre ces deux pôles, un basculement semble s'opérer à la faveur de l'entre-deux-guerres.

La seconde année de séminaire, commencée à la rentrée 2003, a été l'occasion d'orienter le travail dans deux directions: l'approfondissement d'un type de structure particulier et une étude de cas. Mais à la différence de l'année précédente, le séminaire alterne désormais dialogue critique à propos de lectures et présentations de travaux par des chercheurs. L'approfondissement proposé s'est naturellement porté sur le cas des fondations américaines. La construction d'un corps de «réformo-academico-professionnels» a ainsi été analysée selon un double point de vue: tout d'abord, à travers l'histoire générale et le fonctionnement de ces structures depuis les premiers tâtonnements, sous la houlette d'Andrew Carnegie avec la Première Guerre mondiale, jusqu'à la machine de précision progressivement constituée grâce à la fortune d'Henri Ford, à partir de la Seconde Guerre mondiale; puis, à travers l'exemple plus spécifique du travail de Ludovic Tournès sur le réseau des boursiers de la fondation Rockefeller en France entre 1920 et 1970. Pour Pierre-Yves Saunier, il s'est agi de montrer la «mise en place de ce que l'on pourrait appeler un "Chicago consensus" en matière de science, voire de pratique, administrative et gouvernemen-

6. Sur les associations internationales, voir Anne Rasmussen, L'Internationale scientifique 1890-1914, thèse de doctorat en histoire, École des hautes études en sciences sociales, Paris, 1995. Sur les « entrepreneurs », voir Helen Meller, Patrick Geddes : Social Evolutionist and City Planner, Londres, Routledge, 1990 et Ratyward W. Boyd, The Universe of Information : the Work of Paul Otlet for Documentation and International Organisation, Moscou, FID, 1975.

7. Sur la «pensée de Chicago», voir Steven J. Diner, A City and its Universities. Public Policy in Chicago 1892-1919, Chapel Hill, University of North Carolina Press, 1980. Sur les fondations américaines, voir Stanley N. Katz \& Barry D. Karl, «The American Private Philanthropic Foundation and the Public Sphere 1890-1930», dans Minerva, $\mathrm{n}^{\circ} 19,1980$, p. 236-270. 
tale, qui n'est peut être nulle part plus sensible que dans la politique menée par les organismes internationaux dans les années 1950 et 1960, en particulier en direction des pays en développement ».

Depuis quelques séances, le séminaire s'oriente dans une autre direction. Après avoir étudié l' «économie » générale de cette globalisation urbaine, il est proposé aux participants de comprendre comment «vecteurs» et «structures» s'articulent à partir de cas concrets. Une première séance a été consacrée à l'œuvre d'un «entrepreneur » comme John Nolen, une seconde aux «vecteurs» qu'ont pu constituer les expositions de Patrick Geddes qu'étudie Pierre Chabard dans son travail de thèse. Une autre poursuivit ce travail sur les «vecteurs » à partir du cas des congrès internationaux d'architecture moderne, avec l'intervention de Marilena Kourniati. Enfin, un retour sur l'échelle municipale conclura l'année universitaire ${ }^{8}$.

Peu à peu, le séminaire semble atteindre le but qu'il s'était fixé: contribuer à une mise en perspective de la globalisation et faire de cette dernière une production historique. Loin de montrer le visage simple souvent présenté dans le vif débat qui traverse nos sociétés, le séminaire dévoile les aspects contrastés et complexes de cet objet qui reste controversé. À l'heure où les positions politiques évoluent rapidement et où le mirage de la disparition de la souveraineté des États dans un océan mondialisé s'estompe sous le feu de l'actualité, une telle tentative ne peut être que bienvenue.

8. La dernière séance a eu lieu le 3 juin, consacrée aux présentations croisées des travaux de Patrick Leitner (circulations architecturales Paris-New York) et Angelo Bertoni (les milieux réformateurs à Nancy-Luxembourg-Bruxelles). 